\title{
30. CALCAREOUS NANNOFOSSIL AGE DETERMINATIONS LEG 7, DEEP SEA DRILLING PROJECT
}

\author{
Stefan Gartner, Jr., Rosenstiel School of Marine and Atmospheric Sciences, University of Miami, Miami, Florida
}

The data presented here represent age determinations made by means of calcareous nannofossils on sediment cores recovered in the Pacific Ocean during Leg 7 of the Deep Sea Drilling Project. All determinations were made with a light microscope on smear slides prepared from unprocessed samples.

The accuracy of the age determination differs from one sample to the next, owing largely to the differences in assemblages. Assemblages containing age diagnostic species may be assigned to a zone. Some samples, however, contain few or no age diagnostic species; in which case, relatively broader age assignments are made that are commonly equivalent to the interval represented by a stage. Stage terms are avoided (except in the late Miocene), as it seems more reasonable to use such terms as early Miocene or middle Eocene, than to refer to one or another stage term used for the respective intervals.

In no case is an exhaustive species list given for a sample. For the most part only those species are recorded which actually aid in the age determination of a sample. It should be stressed that these results are preliminary, and will be subject to minor revisions as more detailed studies are made.

\section{Hole 62.0}

Sample 7-62.0-1-1, 8-9 cm:

Discoaster brouweri, Discoaster pentaradiatus, Pseudoemiliania lacunosa, Reticulofenestra pseudoumbilica, Sphenolithus abies, Helicopontosphaera kamptneri.

Age: Middle Pliocene (possibly mixed).

Sample 7-62.0-1-6, 8-9 cm:

Sphenolithus abies, Discoaster pentaradiatus, Reticulofenestra pseudoumbilica, Discoaster brouweri, Helicopontosphaera kamptneri, Helicopontosphaera sellii.

Age: Probably early Pliocene.

Sample 7-62.0-2-1, 92-93 cm:

Cyclococcolithina leptopora, Coccolithus pelagicus, Discoaster brouweri, Sphenolithus abies, Discoaster quinqueramus.

Age: Late Miocene (probably Discoaster quinqueramus Zone).

Sample 7-62.0-2-6, 12-13 cm:

Discoaster neohamatus, Sphenolithus abies, Discoaster quinqueramus.

Age: Late Miocene.
Sample 7-62.0-3-1, 8-9 cm:

Sphenolithus abies, Cyclococcolithina leptopora, Discoaster variabilis, Discoaster calcaris, Discoaster quinqueramus, Discoaster hamatus (5 rays), Catinaster coalitus, Reticulofenestra pseudoumbilica.

Age: Late Middle Miocene (Tortonian).

Sample 7-62.0-3-6, 7-8 cm:

As above.

Sample 7-62.0-4-1, 11-12 cm:

Sphenolithus heteromorphus, Coccolithus floridanus.

Age: Early Middle Miocene (probably Helicopontosphaera ampliaperta Zone).

Sample 7-62.0-4-6, 9-10 cm:

As above.

Sample 7-62.0-5-2, 11-12 cm:

Coccolithus floridanus, Sphenolithus moriformis.

Age: Probably Oligocene (assemblage not age diagnostic).

Sample 7-62.0-5-4, 9-10 cm:

As above.

Hole 62.1

Sample 7-62.1-1-2, 28-29 cm:

Gephyrocapsa oceanica, Emiliania huxleyi.

Age: Late Pleistocene-Holocene.

Sample 7-62.1-37-2, 9-10 cm:

Sphenolithus abies. Reticulofenestra pseudoumbilica, aff. Discoaster brouweri, Discoaster exilis, Coccolithus pelagicus.

Age: Middle Miocene.

\section{Hole 63.0}

Sample 7-63.0-1-1, 10-11 cm:

Discoaster brouweri, Gephyrocapsa oceanica, Umbilicosphaera mirabilis.

Age: Late Pleistocene (contaminated).

Sample 7-63.0-1-2, 6-7 cm:

Gephyrocapsa oceanica, Umbilicosphaera mirabilis.

Age: Late Pleistocene. 
Sample 7-63.0-2-2, 9-10 cm:

Sphenolithus abies, Discoaster brouweri, Reticulofenestra pseudoumbilica, Discoaster quinqueramus, Discoaster surculus, Ceratolithus rugosus.

Age: Early Pliocene

Sample 7-63.0-2-6, 8-9 cm:

Discoaster brouweri, Discoaster variabilis, Discoaster surculus, Discoaster quinqueramus, Sphenolithus abies. Age: Uppermost Miocene.

Sample 7-63.0-3-1, $54-55 \mathrm{~cm}$ :

Coccolithus floridanus, Sphenolithus heteromorphus, Coccolithus pelagicus, Discoaster druggi.

Age: Early Middle Miocene (probably Helicopontosphaera ampliaperta Zone).

Sample 7-63.0-3-4, 8-9 cm:

As above.

Sample 7-63.0-4-1, 22-23 cm:

Coccolithus floridanus, Sphenolithus moriformis, Coccolithus pelagicus, Discoaster sp.

Age: Middle Oligocene to early Miocene (assemblage not age diagnostic).

Sample 7-63.0-4-2, 7-8 cm:

Discoaster druggi, Coccolithus floridanus, Sphenolithus moriformis, Coccolithus pelagicus.

Age: Early Miocene.

Sample 7-63.0-5-2, 11-12 cm:

Sphenolithus moriformis, Coccolithus floridanus, Coccolithus pelagicus, Discoaster obtusus, Helicopontosphaera intermedia, cf. Discoaster druggi, Sphenolithus ciperoensis.

Age: Late Oligocene to basal Miocene.

Sample 7-63.0-6-6, 9-10 cm:

Coccolithus floridanus, Sphenolithus predistentus, Sphenolithus moriformis, Sphenolithus distentus.

Age: Early to middle Oligocene (Sphenolithus distentus or Sphenolithus predistentus Zone).

Sample 7-63.0-7-1, 17-18 cm:

Coccolithus floridanus, Sphenolithus predistentus.

Age: Early Oligocene (Sphenolithus predistentus Zone).

Sample 7-63.0-7-6, 11-12 cm:

Coccolithus floridanus, Sphenolithus predistentus.

Age: As above.

Sample 7-63.0-8-3, 11-12 cm:

Coccolithus floridanus, Sphenolithus predistentus, Reticulofenestra scissura, Zygrhablithus bijugatus.

Age: Early Oligocene (lower Sphenolithus predistentus Zone).
Sample 7-63.0-9-4, 6-7 cm:

Coccolithus floridanus, Sphenolithus predistentus, Reticulofenestra scissura.

Age: As above.

\section{Hole 63.1}

Sample 7-63.1-3-2, 8-9 cm:

Discoaster brouweri, Discoaster variabilis, Sphenolithus abies, Discoaster pentaradiatus, Pseudoemiliania lacunosa.

Age: Middle Pliocene (possibly mixed).

Sample 7-63.1-5-1, 59-60 cm:

Discoaster brouweri, Discoaster pentaradiatus, Reticulofenestra pseudoumbilica, Sphenolithus abies.

Age: Early Pliocene.

Sample 7-63.1-14-6, 11-12 cm:

Coccolithus floridamus, Sphenolithus heteromorphus. Age: Early Miocene (probably Helicopontosphaera ampliaperta Zone).

\section{Hole 63.2}

Sample 7-63.2-3-4, 9-10 cm:

Discoaster pentaradiatus, Sphenolithus abies, Reticulofenestra pseudoumbilica, Discoaster surculus, Ceratolithus rugosus.

Age: Early Pliocene.

\section{Hole 64.0}

Sample 7-64.0-1-1, 60-61 cm:

Gephyrocapsa oceanica, Emiliania huxleyi, Umbilicosphaera mirabilis.

Age: Late Pleistocene to Recent.

Sample 7-64.0-1-6, 70-71 cm:

Gephyrocapsa oceanica, Umbilicosphaera mirabilis.

Age: Late Middle Pleistocene.

Sample 7-64.0-2-1, 14-15 cm:

Discoaster pentaradiatus. Pseudoemiliania lacunosa, Sphenolithus abies, Discoaster brouweri, Ceratolithus rugosus, Reticulofenestra pseudoumbilica.

Age: Middle Pliocene.

Sample 7-64.0-2-6, 7-8 cm:

Sphenolithus abies, Reticulofenestra pseudoumbilica, Discoaster brouweri, Discoaster pentaradiatus, Ceratolithus rugosus.

Age: Early to middle Pliocene.

Sample 7-64.0-3-6, 10-11 cm:

Cyclococcolithina leptopora, Sphenolithus abies. Reticulofenestra pseudoumbilica, Discoaster brouweri, Discoaster quinqueramus.

Age: Late Miocene (Messinian). 
Sample 7-64.0-4-6, 80-81 cm:

Reticulofenestra pseudoumbilica, Discoaster hamatus (5 rays), Sphenolithus abies.

Age: Late Middle Miocene (early Tortonian).

Sample 7-64.0-5-6, 12-13 cm:

Sphenolithus heteromorphus, Coccolithus floridanus, Discoaster exilis.

Age: Early Middle Miocene (probably Sphenolithus heteromorphus Zone).

Sample 7-64.0-6-6, 9-10 cm:

Coccolithus floridanus, Discoaster deflandrei, Sphenolithus belemnos.

Age: Early Miocene (Sphenolithus belemnos Zone).

Sample 7-64.0-7-6, 15-16 cm:

Coccolithus floridanus, Coronocyclus nitescense, Sphenolithus moriformis.

Age: Middle Oligocene to early Miocene (assemblage not age diagnostic).

Sample 7-64.0-8-3, 9-10 cm:

Sphenolithus moriformis, Coccolithus floridanus, Sphenolithus ciperoensis.

Age: Late Oligocene (Sphenolithus ciperoensis Zone).

Sample 7-64.0-10-2, 9-10 cm:

Reticulofenestra scissura, Sphenolithus predistentus, Helicopontosphaera compacta, Reticulofenestra umbilica, Lanternithus minutus.

Age: Basal Oligocene (Helicopontosphaera recitulata Zone).

\section{Hole 64.1}

Sample 7-64.1-1-2, 10-11 cm:

Sphenolithus heteromorphus, cf. Discoaster exilis, Coccolithus floridanus, cf. Cyclococcolithus leptoporus.

Age: Middle Miocene (Sphenolithus heteromorphus Zone).

Sample 7-64.1-5-6, 7-8 cm:

Coccolithus floridanus, Sphenolithus moriformis, Sphenolithus heteromorphus.

Age: Middle Miocene (Helicopontosphaera ampliaperta Zone).
Sample 7-64.1-6-4, 10-11 cm:

Coccolithus floridanus, Coronocyclus nitescense, Helicopontosphaera intermedia, aff. Sphenolithus belemnos. Age: Early Miocene (Sphenolithus belemnos Zone).

Sample 7-64.1-7-4, 12-13 cm:

Coccolithus floridanus, Sphenolithus moriformis.

Age: Middle Oligocene to early Miocene (assemblage not age diagnostic).

Sample 7-64.1-8-2, 9-10 cm:

Coccolithus floridanus, Sphenolithus moriformis, Sphenolithus distentus, Sphenolithus ciperoensis.

Age: Middle Oligocene (probably Sphenolithus distentus Zone).

Sample 7-64.1-9-3, 10-11 cm:

Bramletteius serraculoides, Discoaster tani tani, Reticulofenestra umbilica, Cyclococcolithina formosa, Sphenolithus pseudoradians, Discoaster barbadiensis.

Age: Uppermost Upper Eocene.

Sample 7-64.1-10-2, 15-16 cm:

Reticulofenestra umbilica, Reticulofenestra scissura, Cyclococcolithina formosa, Chiasmolithus grandis, Sphenolithus radians, Discoaster tani tani, Discoaster barbadiensis, Chiasmolithus solitus.

Age: Late Middle Miocene.

\section{Hole 65.0}

Sample 7-65.0-14-4, 140-141 cm:

Reticulofenestra scissura, Discoaster tani tani, Reticulofenestra umbilica, Coccolithus marismontium, Sphenolithus radians, Cyclococcolithina formosa, Discoaster barbadiensis.

Age: Middle Eocene.

Sample 7-65.0-14-6, 115-116 cm:

Reticulofenestra scissura, Bramletteius serraculoides, Reticulofenestra umbilica, cf. Discoaster, mirus, Cyclococcolithina formosa, Discoaster barbadiensis, cf. Helicopontosphaera compacta.

Age: Late Middle Eocene (this sample may be mixed or contaminated). 